\title{
Editorial
}

\section{Advancing the knowledge base on work transitions in the 21 st century}

\author{
Lynn Shaw ${ }^{\mathrm{a}, *}$ and Karen Jacobs ${ }^{\mathrm{b}}$ \\ a School of Occupational Therapy, The University of Western Ontario, London, ON, Canada \\ ${ }^{\mathrm{b}}$ Boston University, Sargent College of Health and Rehabilitation Sciences, Boston, MA, USA
}

\section{Introduction}

This special issue has two exciting sections. The first has an international focus on advancing knowledge and research on work transitions into the $21^{\text {st }}$ century. The second is a special issue on European Perspectives on work-related education practice and research. The first section of the journal issue is dedicated to the documentation of the outcomes from an international workshop held on work transitions in the summer of 2009 that was titled Work Transitions in the $21^{\text {st }}$ Century: Advancing Occupational Justice. Four research papers and a summary article on the workshop are included.

\section{Work transitions in the 21st Century: Advancing Occupational Justice}

The first paper is the summary of the workshop and the results of the efforts of over 30 participants including the international moderators, presenters, researchers, graduate students and community partners. Dr. Lynn Shaw was the principal investigator on the Social Sciences and Humanities Research Council of Canada grant that partially supported the workshop (funding was also provided by the School of Occupa-

*Address for correspondence: Lynn Shaw, 1201 Western Rd., Elborn College Room 2542, School of Occupational Therapy, The University of Western Ontario, London, ON, N6G 1H1, Canada. tional Therapy at The University of Western Ontario) along with four co-applicants, Dr. Debbie Rudman, Dr. Lilian Magahles, Dr. Angie Mandich and Suzanne Huot. The first paper is a multi co-authored paper recognizing all who participated contributed to the collective knowledge and workshop outcomes. The Shaw et al. paper overviews the occupational justice perspective that framed the presentations and the workshop activities that culminated in two imperatives and six recommendations for research. Four papers from presentations provide clear examples that supported the development of new directions in research.

Dr. Karen Rebeiro Gruhl offers an invigorating paper on the breadth of inequities tensions and barriers to employment participant for persons with serious mental health illness in Northern Ontario. Her paper underscores the tensions created by policies that are ineffective and are inadequately resourced to support the people they are meant to support. She also uses an appropriate and rigorous methodology based on a multiple method case study design to extract and reveal the source of the tensions. In the paper by Dr. Clare Hocking she examines the sources of barriers that limit access to work and the maintenance of work by reviewing 11 articles with different cases and contexts. Most are consistent with many of the sources of barriers that lead to occupational deprivation as posited by Dr. Ann Wilcox. Both system and structural issues are discussed that can be targeted for change.

Dr. Rosemary Lysaght and colleagues, provide an indepth review of the literature to address the challenge 
for employment for persons with intellectual disabilities (ID). This candid review of theories and real life practices highlights the potential for persons with ID to participate in employment through system and structural changes. In turn, this paper will inform solutions that might work to provide opportunities for persons with ID to be productive citizens in our society. The paper by Dr. Leyshon and Shaw focuses on the need for research with stakeholders and to invite them to have a voice in how return to work might be evaluated. The outcome of this study reflects the processes and the dimensions of returning to work that are relevant to workers and others involved in the transition back to work. The use of concept mapping research is a relatively new research method that included multiple stakeholders who hold traditional divergent views on return to work in a process of working together to define what constitutes work success.

What is common across all four papers is that the focus has moved from considering obtaining employment as the end of the return to work process to a focus that includes maintaining employment. This extension of the process is a key step forward in the study of work transitions in that we can no longer consider resumption of or access to employment the end point. Being able to maintain work participation for persons of difference is part of being and becoming a full citizen in society and the resources and supports that are needed to maintain work in a context where work is constantly transforming must be considered [1]. Maintaining work needs to become part of the discourse in research and practice. Furthermore, using occupational justice as a framework has opened the door to discovery of what are ways that systems and structures have a dialectical relationship that can perpetuate work disparities. These insights in turn point to new avenues for change. Moreover, what we have learned through using an occupational justice perspective is that it brings new potential to the knowledge base that can direct our focus on aspects of the systems, processes, and structures. This perspective then widens and adds to the circle of known traditional employment solutions such as ergonomics or accommodation or assistive devices and work rehabilitation programs. Solutions that can address system and policy tensions can become powerful catalysts of change needed to support successful work transitions. One final observation from these papers and the workshop is that policies and systems that govern resources to support work transition programs require ongoing monitoring and evaluation. Thus, researchers and community members also need to continually evaluate the effectiveness of policies in practice on employment participation and advocate for improving the capacity of the structures to achieve not only access to work, but also the maintenance of employment as part of a person's work trajectory.

Guest Editorial

Lynn Shaw and Karen Jacobs

\section{Reference}

[1] Galheigo, S.M. (2011). What needs to be done? Occupational therapy responsibilities and challenges regarding human rights. Australian Occupational Therapy Journal 58 (2), 60-66. 